Letter to the Editor

\title{
Intractable Restless Legs Syndrome - Look for Other Causes
}

\section{Dear Editor,}

Restless legs syndrome (RLS)—or Willis-Ekbom disease ${ }^{1}$ - is defined as a strong urge to move the legs that is accompanied by uncomfortable and unpleasant sensations. The symptoms are relieved by movementsuch as walking or moving the legs - and are not attributed to any underlying medical or psychological condition. The condition, which is irritating and can severely diminish quality of life, can be intractable. The mainstay of treatment includes calcium channel alpha-2-delta antagonists - such as gabapentin — or dopaminergic agents like dopa agonists and levodopa.

\section{Case Presentation}

A 48-year-old man presented 17 years ago with formication (sensation of crawling ants) on his soles for 3 years. Initially, the symptoms occurred during the day when he was at rest, but soon progressed to affect his sleep at night, leading to diminished sleep quality and wakefulness. He did not report any pain, sensory loss or weakness. The discomfort was only relieved by ambulation and movement of legs. He did not report any significant family or medical history.

Prior to presentation, he consulted an orthopaedic surgeon for neck pain, and magnetic resonance imaging (MRI) of the spine (Figs. 1A and 2A) showed mild cervical spondylosis at the level of C5-6 with thecal - but no significant cordindentation and absence of myelopathic changes. Findings of the neurophysiologic examination were normal, including nerve conduction and electromyography. Somatosensory evoked response and transcranial magnetic stimulation studies were not performed. Sleep study was not completed, as leg discomfort prevented sleep. As he met the diagnostic criteria for RLS, ${ }^{2}$ he was diagnosed with chronic-persistent RLS. Differential diagnoses of sensory polyneuropathy and akathisia were excluded by normal neurophysiology and absence of previous neuroleptic exposure.

Initially, he was given levodopa-benserazide (madopar) $125 \mathrm{mg}$ nocte and clonazepam $1 \mathrm{mg}$ once daily (OD), but the symptoms persisted despite upward titration of medications. At 3 years, he was diagnosed with reactive depression and treated with diazepam $2 \mathrm{mg}$ and amitriptyline $10 \mathrm{mg}$ OD. Further investigations revealed microcytic hypochromic anaemia from iron deficiency (haemoglobin, $12.8 \mathrm{~g} / \mathrm{dL}$; serum ferritin $<1 \mu \mathrm{g} / \mathrm{L}$; and serum iron, $6.5 \mu \mathrm{mol} / \mathrm{L}$ ) which was a possible secondary cause of RLS. ${ }^{2} \mathrm{He}$ was given iron replacement therapy, but RLS symptoms persisted despite normalisation of laboratory parameters (serum ferritin, $9.1 \mu \mathrm{g} / \mathrm{L}$; and serum iron, $16.6 \mu \mathrm{mol} / \mathrm{L})$.

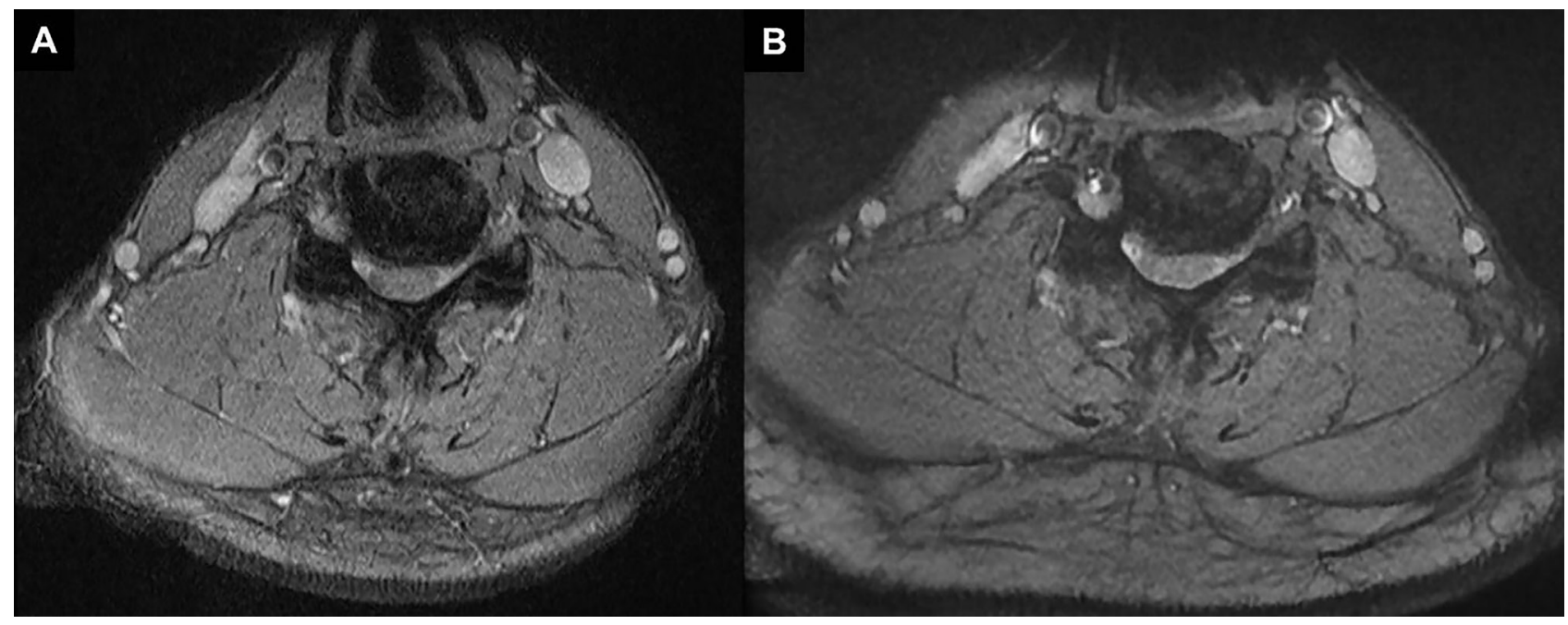

Fig. 1. A: Axial magnetic resonance image (MRI) of spine showed mild thecal and central cord indentation at the level of C5-6. B: Subsequent axial MRI showed myelopathic changes with more severe central cord indentation at C5. 


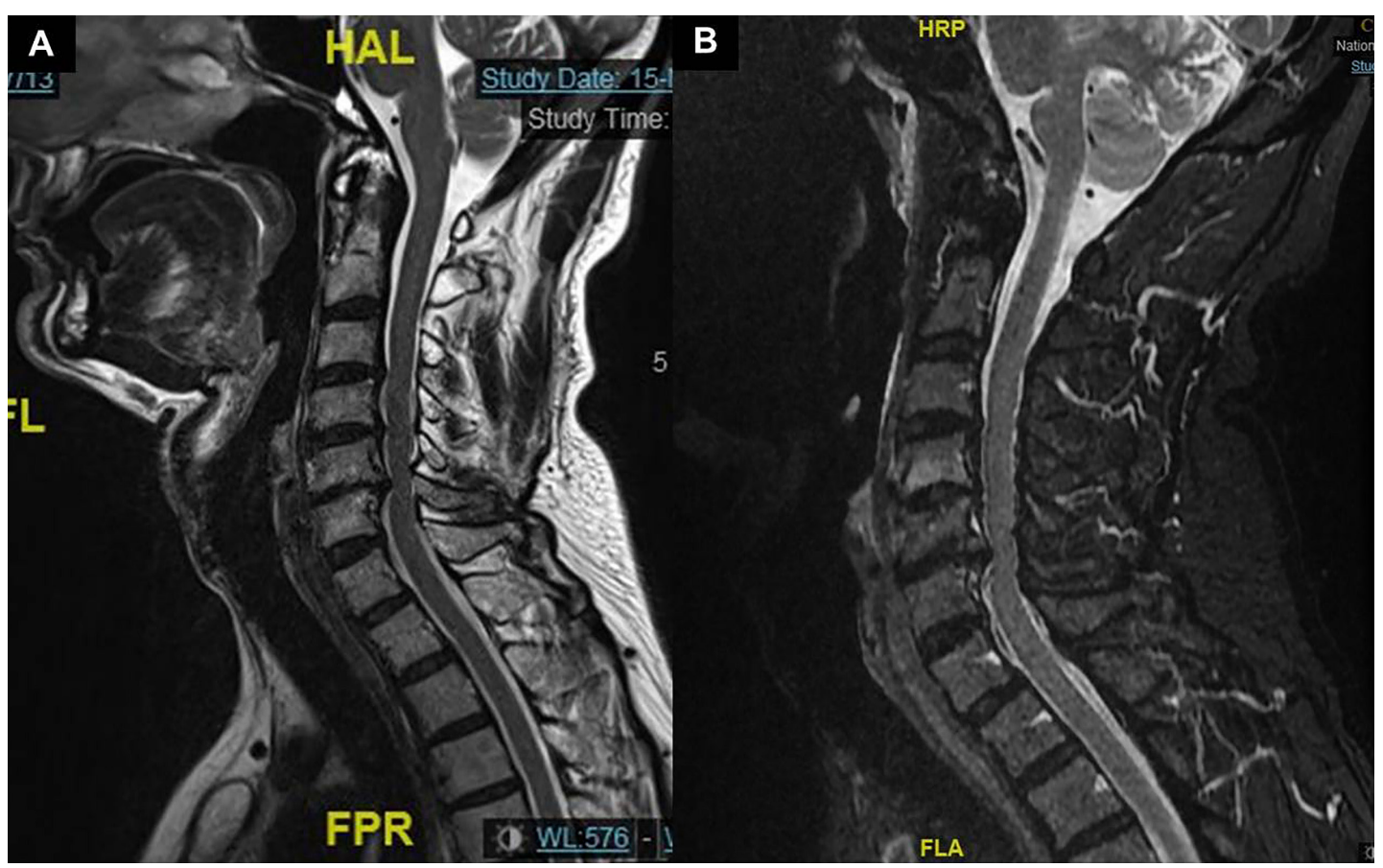

Fig. 2. Intial (A) and subsequent (B) sagittal magnetic resonance images of spine showed mild thecal and central cord indentation at level of C5-6 that worsens progressively with the development of upper motor neuron signs.

Over 11 years, he was given various medicationsbenzodiazepines, calcium channel alpha-2-delta antagonists, catechol-o-methyl transferase inhibitors, dopa agonists, levodopa, neuroleptics, opioids and tricyclic antidepressants - to treat formication and intractable, severe RLS. Eventually, he responded to a combination oflevodopabenserazide $250 \mathrm{mg} 4$ times a day, entacapone $200 \mathrm{mg}$ twice daily and midazolam $15 \mathrm{mg}$ nocte, which helped him to sleep better. Attempts to wean him off benzodiazepines and lower the dose of levodopa-benserazide were ineffective. He lost his job and the depression and panic attacks worsened. In 2001, he was admitted after overdosing on an unknown antidepressant in a suicide attempt. He resumed followup with the psychiatrist and his mood improved after he learned to manage his condition.

After initial diagnosis and treatment for RLS, he was referred to our clinic 11 years later. Physical examination revealed hypertonia in both upper and lower limbs, hyperreflexia in left upper limb, a right inverted supinator reflex and extensor plantar responses bilaterally. He experienced full motor power (Medical Research Council grade 5/5), but sensory loss on pinprick and temperature up to the $5^{\text {th }}$ cervical dermatome. The cervical spine MRI showed myelopathic changes and multilevel cervical spondylosis that was worst at the level of C5-6 with severe central canal stenosis and cord signal changes (Figs. 1B and 2B).

The atypical features - abnormal findings on cervical spine MRI, failure to respond to medications and presence of RLS symptoms during the day-indicated secondary RLS or cervical myelopathy that mimicked RLS. As surgical intervention could alleviate his symptoms and confirm cervical myelopathy as the cause of RLS-like symptoms, he was referred to the neurosurgeon who recommended decompression laminectomy, since further aggravation of cervical cord compression could potentially cause catastrophic quadriplegia. However, he declined surgery and further investigation with transcranial magnetic stimulation, and opted for follow-up and medical treatment with the neurologist and neurosurgeon, even though his quality of life was badly affected by RLS; this was seen in his low score of $\leq 25$ on 6 of 8 items and $\leq 50$ in 1 of 8 measures in the 36-item Short Form Health Survey. These low scores indicated role limitations due to physical health and emotional problems, energy and fatigue, emotional well-being, social functioning and pain.

\section{Discussion}

RLS is classified as primary (idiopathic) or secondary (associated with end-stage renal disease, iron deficiency, Parkinson's disease, polyneuropathies, pregnancy or rheumatoid arthritis). ${ }^{2}$ RLS prevalence ranges from 5-15\% in the general population, ${ }^{2}$ most of whom have primary RLS. The aetiology of RLS has been attributed to spinal cord injury; in 1 study, ${ }^{3}$ up to $17.9 \%$ of patients with the injury presented with RLS. In the literature, there are case reports of multiple sclerosis, traumatic myelopathy and 
spinal cord infarction that caused RLS, but only 1 case of spondylotic cervical myelopathy was reported to have caused RLS. ${ }^{3}$

Our patient initially met the diagnostic criteria of primary RLS; however, signs of secondary RLS were missed in him. The normal results in his nerve conduction studies and electromyogram could have contributed to this oversight. Unfortunately, somatosensory evoked responses - which could have yielded a clue on underlying myelopathywere not assessed. It is unlikely that iron deficiency had caused secondary RLS, since the symptoms persisted after anaemia resolved and iron levels had normalised.

In our patient, the onset of upper motor neuron (UMN) signs that were consistent with C5 myelopathy informed the diagnosis of secondary RLS from cervical myelopathy (Fig. 3). The initial lack of UMN signs could be attributed to compression of only the spinothalamic tracts ${ }^{4}$ - but not the pyramidal tracts (Fig. 3C) - even though thecal and cord compression was seen in earlier MRI. Worsening cord compression that affected the anterior horn cells and corticospinal tracts (Fig. 3D) then resulted in the UMN signs that were consistent with C5 myelopathy.

It is not possible to know, for certain, whether our patient had primary RLS with concurrent cervical myelopathy or cervical myelopathy that is coincidental and unrelated to RLS. Had he opted for decompression laminectomy, complete or significant reduction of his symptoms would have confirmed cervical myelopathy as the likely cause of RLS. He also declined transcranial magnetic stimulation, which studies conduction in the descending corticospinal tract of the spinal cord. Had he been found to have prolonged central conduction time in the upper limbs and diminished motor evoked potentials - which predict better surgical outcome ${ }^{5}$ - a more forceful case for decompression laminectomy could have been made. As it is, he declined surgical intervention as he was fearful of the risk of quadriparesis and spasticity associated with cervical decompression laminectomy, even after he
A

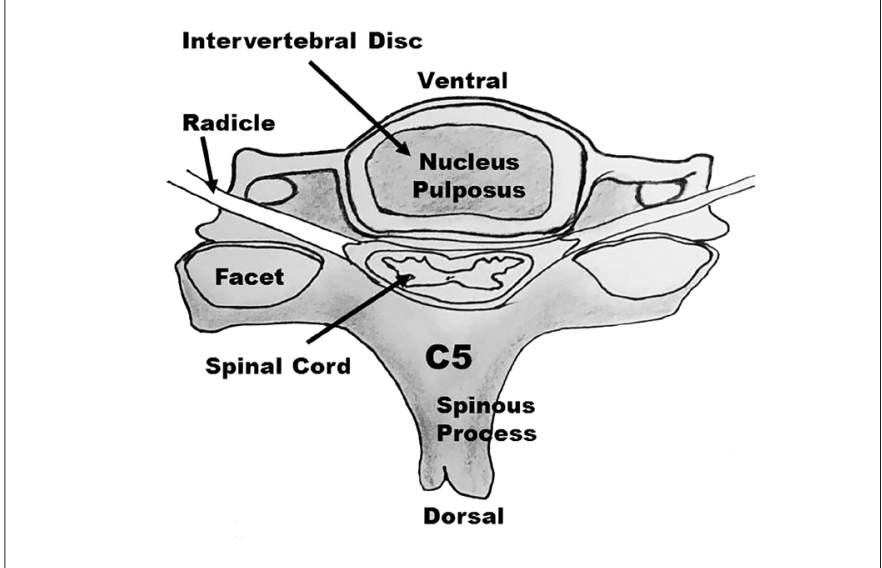

C

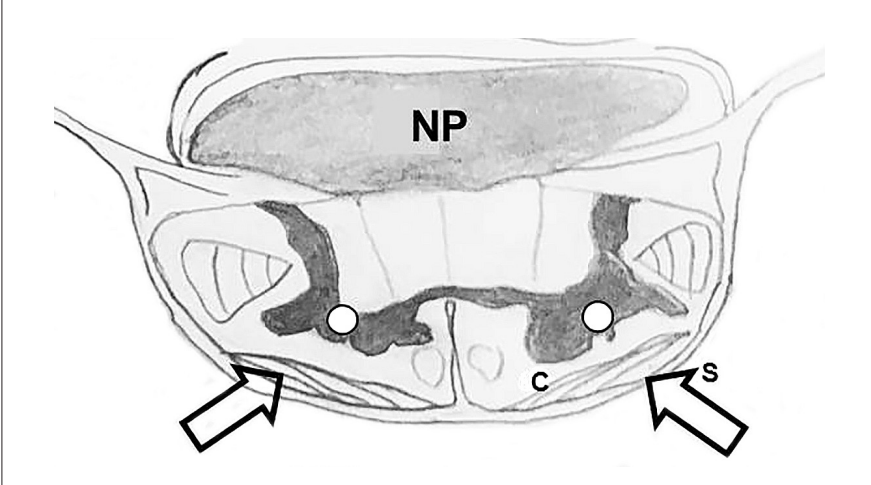

B

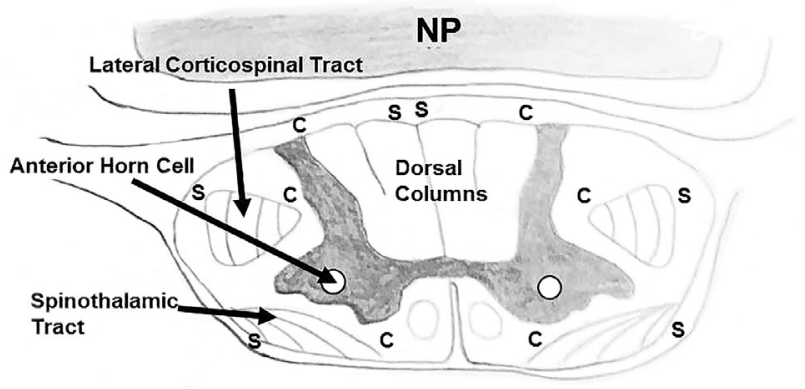

D

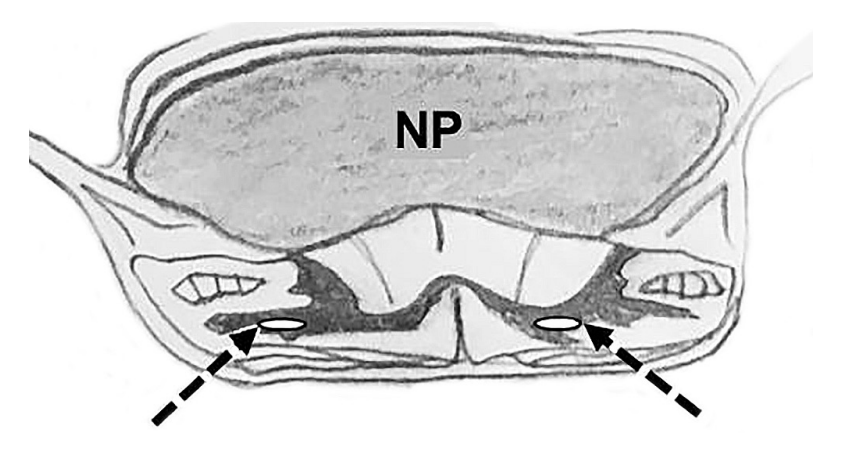

Fig. 3. Progression of disc herniation at C5-6 caused somatotopic symptoms initially, mimicking RLS, before the development of upper motor neuron (UMN) signs 17 years later. A: Normal vertebral and spinal cord structure. B: Enlarged diagrammatic representation of the normal spinal cord. Note the somatotopic representation within the spinothalamic tracts with the sacral (S) and lumbar tracts located more laterally, and the cervical (C) and thoracic tracts located more medially. C: Herniation of interverterbral disc at C5 caused compression of the caudal (lumbosacral) spinothalamic tracts (hollow arrows) that produced the initial RLS symptoms, but not the (circular-shaped) anterior horn cells or corticospinal tracts. D: As cord compression from disc herniation progressed, the anterior horn cells (now flattened) and corticospinal tracts were compressed (dashed arrows), leading to onset of UMN signs. NP: Nucleus pulposus 
was informed that he may experience $20-60 \%$ risk of neurological deterioration between 3 to 6 years without surgical decompression. ${ }^{6}$

In RLS patients, there is increased prevalence of comorbid psychiatric conditions - such as anxiety and depression-even in those who are on treatment. ${ }^{7}$ Our patient remains on psychiatric follow-up and treatment for anxiety and depression. RLS patients also experienced lower quality of life, poorer work productivity and more sleep disturbances. ${ }^{7}$

This case underscores the importance of accurate diagnosis and appropriate treatment. Although he expressed satisfaction with current treatment and medication regimen, ${ }^{2}$ the diagnosis of secondary RLS attributable to cervical myelopathy raises questions about the appropriateness of treatment with analgesics, benzodiazepines and levodopa-benserazide. Although dopa agonists were preferred to levodopa as first-line treatment of RLS - levodopa is known to cause rebound or augmentation of RLS symptoms ${ }^{2}$ - they were poorly tolerated in the patient. Likewise, calcium channel alpha2-delta antagonists such as gabapentin also failed to bring about relief in him.

After he was diagnosed with secondary RLS, attempts were made to wean him off levodopa and midazolam since there were concerns about potential neurotoxicity (generation of reactive oxygen species were seen in Parkinson's disease patients), lack of evidence in treating myelopathic pain with levodopa ${ }^{8}$ and likelihood of addiction to midazolam. ${ }^{9}$ However, he insisted that a lower dose of both drugs exacerbated his formication and he vehemently opposed all attempts to decrease or wean him off them. His management is further complicated by treatment for his depression, since the use of amitriptyline and benzodiazepines with anticonvulsants, antidepressants, dopamine antagonists, neuroleptics and serotonergic reuptake inhibitors can exacerbate RLS symptoms. ${ }^{2}$

\section{Conclusion}

This case illustrates the difficulty of managing RLS. It is also important to exclude secondary causes of lower limb restlessness. RLS remains a difficult condition to treat and it can cause physical discomfort and mental distress. However, effective treatment can alleviate symptoms and greatly improve quality of life in RLS patients.

\section{REFERENCES}

1. Gonzalez-Latapi P, Malkani R. Update on restless legs syndrome: from mechanisms to treatment. Curr Neurol Neurosci Rep 2019;19:54.

2. Wijemanne S, Ondo W. Restless legs syndrome: clinical features, diagnosis and a practical approach to management. Pract Neurol 2017;17:444-52.

3. Kumru H, Vidal J, Benito J, Barrio M, Portell E, Valles M, et al. Restless leg syndrome in patients with spinal cord injury. Parkinsonism Relat Disord 2015;21:1461-4.

4. Tings T, Baier PC, Paulus W, Trenkwalder C. Restless legs syndrome induced by impairment of sensory spinal pathways. J Neurol 2003;250:499-500.

5. Lo YL. The role of electrophysiology in the diagnosis and management of cervical spondylotic myelopathy. Ann Acad Med Singapore 2007;36:886-93.

6. Fehlings MG, Wilson JR, Yoon ST, Rhee JM, Shamji MF, Lawrence BD. Symptomatic progression of cervical myelopathy and the role of nonsurgical management: a consensus statement. Spine 2013;38:S19-20.

7. Svetel MV, Jovic JS, Pekmezovic TD, Kostic VS. Quality of life in patients with primary restless leg syndrome: community-based study. Neurol Sci 2015;36:1345-51.

8. Lim E. A walk through the management of Parkinson's disease. Ann Acad Med Singapore 2005;34:188-95.

9. Ng WL, Mythily S, Song G, Chan YH, Winslow M. Concomitant use of midazolam and buprenorphine and its implications among drug users in Singapore. Ann Acad Med Singapore 2007;36:774-7.

Shi Ni Tan, ${ }^{1,2}$ MBBS (Singapore),

Erle CH Lim, ${ }^{1}$ MMed (Int Med), FRCPS (Glasgow)

${ }^{1}$ Division of Neurology, University Medicine Cluster, National University Hospital, Singapore

${ }^{2}$ Ministry of Health Holdings, Singapore

Address for Correspondence: Dr Tan Shi Ni, c/o Division of Neurology, University Medicine Cluster, National University Hospital, 1E Kent Ridge Road, Level 10, NUHS Tower Block, Singapore 119228.

Email: tanshini95@gmail.com 